\title{
A 4-quadrant Curvelet Transform for Denoising Digital Images
}

\author{
$\begin{array}{ll}\text { P. K. Parlewar } & \text { K. M. Bhurchandi } \\ & \end{array}$ \\ ${ }^{1}$ Department of Electronic and Communication Engineering, Ramdeobaba College of Engineering and Management, Nagpur, India \\ ${ }^{2}$ Department of Electronic Engineering, Visvesvaraya National Institute of Technology, Nagpur, India
}

\begin{abstract}
The conventional discrete wavelet transform (DWT) introduces artifacts during denoising of images containing smooth curves. Finite ridgelet transform (FRIT) solved this problem by mapping the curves in terms of small curved ridges. However, blind application of FRIT all over an image is computationally heavy. Finite curvelet transform (FCT) selectively applies FRIT only to the tiles containing small portions of a curve. In this work, a novel curvelet transform named as 4-quadrant finite curvelet transform (4QFCT) based on a new concept of 4-quadrant finite ridgelet transform (4QFRIT) has been proposed. An image is band pass filtered and the high frequency bands are divided into small non-overlapping square tiles. The 4QFRIT is applied to the tiles containing at least one curve element. Unlike FRIT, the 4QFRIT takes 4 sets of radon projections in all the 4 quadrants and then averages them in time and frequency domains after denoising. The proposed algorithm is extensively tested and benchmarked for denoising of images with Gaussian noise using mean squared error (MSE) and peak signal to noise ratio (PSNR). The results confirm that 4QFCT yields consistently better denoising performance quantitatively and visually.
\end{abstract}

Keywords: Curvelet transform, ridgelet transform, 4-quadrant ridgelet transform, 4-quadrant curvelet transform, denoising.

\section{Introduction}

The visual images should be smooth or spontaneous on regions and curves. They should also be sharp enough to protect high frequency information. Every electronic unit that sends or receives an image signal is susceptible to noise. One may not visually observe the noise when he looks at an image. However, it is always corrupted with noise to different extents. Thus, denoising is a very important field of image processing which aims to improve the visual quality of digital images by minimizing noise contents.

Wavelet theory has been studied for a fairly long time and has applications in many engineering fields. The basic wavelet theory has been proposed and customized for various applications such as histogram operations ${ }^{[1]}$, watermarking ${ }^{[2]}$, medical image analysis ${ }^{[3-5]}$, face recognition ${ }^{[6-7]}$, fingerprint recognition ${ }^{[8]}$, etc. While using it for compression, coding or even as a denoising tool for images containing smooth curves, it introduces artifacts. Though it has this limitation while processing curved edges, it is being widely used for image compression and coding. Denoising performance of multi-scale Bayesian approaches $^{[9]}$ and wavelets are presented ${ }^{[10]}$. Starck et al. ${ }^{[11]}$ proved that a new approach named "Ridgelets" performs better than the mature wavelets for processing curves. Subsequently, finite ridgelet transform (FRIT) was presented and explored further for denoising of images containing curves. It has been observed that FRIT introduces less artifacts compared to discrete wavelet transform (DWT) during processing of curves, as it processes images using polar representation system like radon projections. Keeping aside the denoising performance, it has been observed that the curves processed by FRIT still contain some artifacts.

Finite curvelet transform (FCT) is a new multi-scale geo-

Manuscript received January 10, 2012; revised October 23, 2012 metric function introduced for processing images containing objects which are smooth and continuous across curves ${ }^{[11]}$. The FCT is designed to represent edges and other singularities along the curves much efficiently than the other orthogonal transforms ${ }^{[8,10-12]}$. The length and width of a ridge at a fine scale are related by scaling law: width $\approx$ length $^{2}$. So anisotropy increases with decreasing scale like a power law. On an image with successively twice differentiable singularities, non-optimal systems like Fourier and wavelets have approximation errors presented in (1) and $(2)^{[10-13]}$. If $m$ represents the number of coefficients used for approximation of a signal, $f$ and $f_{m}$ are the approximated forms using Fourier $(F)$ and Wavelet $(W)$ approximations.

$$
\begin{aligned}
& \lim _{m \rightarrow \infty}\left\|f-\hat{f}_{m}^{F}\right\|=m^{-\frac{1}{2}} \\
& \lim _{m \rightarrow \infty}\left\|f-\hat{f}_{m}^{W}\right\|=m^{-1} .
\end{aligned}
$$

The FRIT employs a set of radon projections to process images using a system similar to polar coordinates. In this system, the curves are mapped in terms of ridges, i.e., small horse shoe shaped curves rather than line segments. Thus, there is a reasonable scope to believe that image curves will now be better processed as compared to wavelets. However, computation complexity of FRIT of an image is very heavy due to computations of projections. As many parts of an image may not contain any curve, this increase in computation overhead is undue. The FRIT is applied only on small square size non-overlapping tiles of the high frequency bands of the image containing curved edges. The FCT is thus expected to yield better results as the tiles without edges are processed using the rectangular coordinate system like wavelets, and the tiles containing curved edges are processed using polar coordinate systems like ridgelets. Thus, the FCT requires much less computations compared to FRIT. Usually, smaller prime number tile sizes like $3 \times 3$ 
or $5 \times 5$ are used for better processing of sharp curves using FRIT. Thus, FCT is a hybrid algorithm that uses DWT for tiles without curves and FRIT for tiles including curves to yield curvelet coefficients. The coefficients are further denoised followed by inverse computations for a denoised reconstruction. The approximation error while in representing a curve with $C$ singularities using FCT is presented in (3). It is obvious that the approximation error is less as compared to Fourier and wavelets.

$$
\lim _{m \rightarrow \infty}\left\|f-\hat{f}_{m}^{C}\right\|=C m^{-2}(\log m)^{3} .
$$

The approximation error in (3) is roughly $m^{-2[10-12]}$. From (3), the FCT offers the closest $m$-term approximation to the lower bound ${ }^{[2,3,5,14,15]}$. Therefore, in images containing large number of curves with small radii of curvatures, it would be advantageous to use curvelet algorithm for better processing of curves due to its processing capabilities in planar and polar coordinate systems. Structural elements of curvelet transform include dimensions, location and orientation ${ }^{[16]}$, which offer it good orientation characteristics. Thus, FCT maintains original smoothness of the curved edges while denoising images compared to DWT that introduces artifacts. This has already attracted good research efforts and yielded better results in image denoising ${ }^{[8,12,13,17-22]}$ and curve representation.

In this paper, we propose a 4-quadrant curvelet transform (4QFCT) for better curve representation and denoising of digital images compared to the published curvelet transform. The 4QFCT uses a novel 4-quadrant finite ridgelet transform (4QFRIT) that computes 4 sets of projections in the 4 quadrants for the tiles containing curves. Averaging of the curvelet coefficients leads to the first level of denoising. Denoised image is reconstructed as the average of the 4 individual reconstructions. The mean squared error (MSE) or peak signal to noise ratio (PSNR) show that the 4QFCT yields better denoising performance. The result images show that all the edges and curves are reconstructed more smoothly than the FRIT and FCT transform.

The organization of this paper is as follows. Section 2 describes the concepts required for building the proposed transform. Section 3 introduces the novel concept of 4QFRIT, followed by 4QFCT in Section 4. Experimental procedures are discussed in Section 5, and the new transform is qualitatively and procedurally compared with the other contemporaries, especially FRIT and FCT. Section 5 benchmarks the results of denoising and curves processing using 4QFCT with the other techniques in presence of varying Gaussian noise. Section 6 concludes the paper.

\section{Basic concepts of curvelet transform}

\section{$2.1 \quad$ Ridgelet transform}

Ridgelet transform is computed by integrating along lines perpendicular to a line that is at an angle $\theta$ with $x$ axis all over a $2 \mathrm{D}$ signal. The continuous ridgelet transform (CRIT) of a function $f(x, y)$ is presented in (4)

$$
\operatorname{CRIT}_{f}(a, b, \theta)=\int \psi_{a, b, \theta}(x, y) \times f(x, y) \mathrm{d} x
$$

where the function $\psi$ is given by

$$
\psi_{a, b, \theta}(x, y)=\frac{1}{\sqrt{a}} \times \frac{y \cos \theta+x \sin \theta-b}{a}
$$

and $y \cos \theta+x \sin \theta=k$.

Therefore, substituting $k$, we get

$$
\psi_{a, b, \theta}(x)=\frac{1}{\sqrt{a}} \times \frac{k-b}{a} .
$$

The parameters $a, b$ and $\theta$ represent scale, location and orientation parameters. The " $k$ " is a constant which defines a projection line. Thus, (6) represents a basic ridge. The ridgelet coefficients are calculated using 1D DWT of rows of radon transform. Most of the image processing algorithms work on point singularities while ridgelet transform is expected to operate on line singularities at an angle $\theta$. This problem is handled using finite radon transform projections $^{[23,25]}$. FRIT is the sampled form of CRIT. The computational complexity of FRIT all over the projection lines of a $2 \mathrm{D}$ signal is very high. But the increased computational load is the cost paid for smoother representation of curved edges in terms of small horse shoe like ridges using radial (polar system like) radon projections. This is the basic advantage of the ridgelet transform and curvelet transform that uses the ridgelet transform on image subband tiles.

\subsection{Curvelet transform}

Curvelet transform developed by Struck et al. ${ }^{[11]}$ is a new multiscale approach to represent edges and other singularities along curves much more efficiently than the traditional transforms using fewer coefficients for a given accuracy of reconstruction. The curvelet transform uses ridgelet transform on areas of a $2 \mathrm{D}$ signal containing curves. The input image is band pass filtered. The curves in the high frequency sub-bands of the filtered image are processed by first separating the image into series of disjoint tiles, and then, analyzing each tile using ridgelet transform separately, if it contains curve ${ }^{[24,26]}$.

The implementation of FCT is described in terms of steps for decomposition and reconstruction. Fig. 1 graphically presents steps of Algorithm 1.

Algorithm 1. The curvelet transform

Step 1. An input image $I$ is filtered into sub-bands, $H_{0}\left(\Delta_{m}, m \geqslant 0\right)$. $H_{0}$ represents low pass component while $\Delta_{m}$ represents high frequency sub-band components.

$$
I \rightarrow\left(H_{0} I, \Delta_{1} I, \Delta_{2} I, \cdots\right) .
$$

Step 2. Each high frequency sub-band is partitioned using a window $w_{Q}$ into $m$ square tiles " $Q$ " $\left(Q_{1}, Q_{2}, \cdots, Q_{m}\right)$. The tiles must have prime number square size.

$$
\Delta_{m} I \rightarrow\left(w_{Q} \Delta_{m} I\right)_{Q \in Q_{m}}
$$

Step 3. The resulting tiles in Step 2 are normalized to obtain $g_{Q}$.

$$
g_{Q}=T_{Q}^{-1} w_{Q} \Delta_{m} I, \quad Q \in Q_{m}
$$

where $T_{Q}=\sum w_{Q} \Delta_{m} I, m \geqslant 0$. 
Step 4. FRIT is applied to each tile $g_{Q}$ with at least one curve element. FRIT generates a set of coefficients $\alpha_{\mu}$ using orthonormal basis elements $\rho_{\lambda}$. This is repeated for all $Q$. $\alpha_{\mu}$ is further used for computing inverse of the ridgelet coefficients $\left(R^{2}\right)$ using a $2 \mathrm{D}$ inverse ridgelet transformation $\left(L^{2}\right)$.

$$
\alpha_{\mu}=\left\langle g_{Q}, \rho_{\lambda}\right\rangle, \mu=(Q, \lambda) \text { for } L^{2} R^{2} .
$$
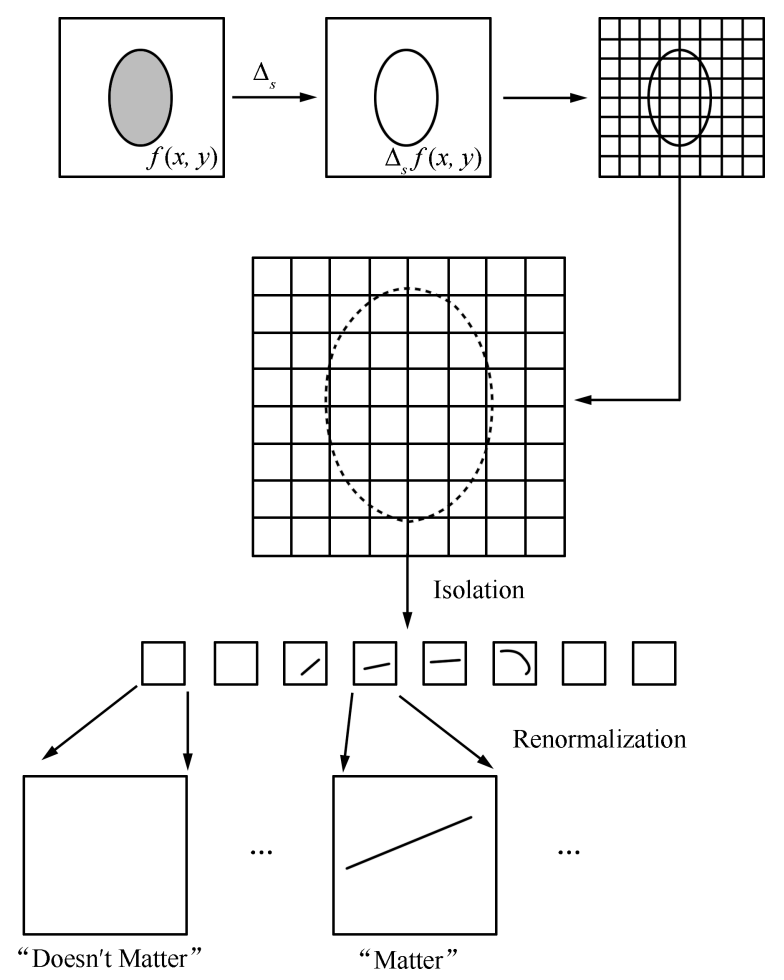

Fig. 1 Organization of finite curvelet transform

Algorithm 2. Reconstruction of original image $^{[17,24]}$

Step 1. Each square is reconstructed from the orthonormal ridgelet system as described using (1).

$$
g_{Q}=\sum_{\lambda} \alpha \lambda Q \rho_{\lambda}
$$

Step 2. Each square available in the previous stage is renormalized to its own proper size using (12).

$$
h_{Q}=T_{Q} g_{Q}, \quad Q \in Q_{m} .
$$

Step 3. The windowing dissection of the windows reconstructed in the previous stage is reversed as in (13).

$$
\Delta_{m} I=\sum_{Q \in Q_{m}} w_{Q} h_{Q}
$$

Step 4. Reconstruct $I$ using (14)

$$
I=H_{0}\left(H_{0} I\right)+\sum_{m>0} \Delta_{m}\left(\Delta_{m} I\right) .
$$

\section{4-quadrant ridgelet transform}

Multiple sets of projections can be used for better reconstruction $^{[27]}$. It is obvious that curves may be better represented in polar coordinates than Cartesian system. Thus, curves are better processed using ridges of the FRIT. This is the initial motivation behind proposing the 4 QFCT. Svalbe and Kingston ${ }^{[28]}$ proved that all Farey sequence points in Cartesian domain cannot be represented in projection domain, specially in the annular or corner regions opposite origin as shown in Figs. 2 (a) and 2 (b). As a result of radial projections, pixels in the annular corner region diagonally opposite to origin are poorly represented in projection domain and may result into introduction of more artifacts in curved edges in that region. The conventional ridgelet transform ${ }^{[29]}$ for denoising works only on the first quadrant as in Fig. 2 (a) for a tile of $11 \times 11$ size. This results in non-uniform representation over the tile in the radial projection domain as observed in Figs. 2 (a). Fig. 2 (b) presents the same concept for the second quadrant. The projections in terms of pixels for the remaining two quadrants of a tile can be easily imagined on the lines of Figs. 2 (a) and 2 (b). The reference point or origin of each quadrant is marked " $O$ " while the most poorly represented corner has been marked " $C$ ". The following digits in Fig. 3 indicate the respective quadrant number. This problem is fairly solved by averaging the 4 quadrant projections over the tile as presented in Figs. 3 (a) and 3(b).

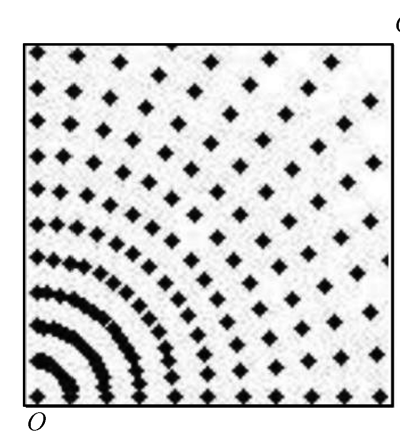

(a) Quadrant 1

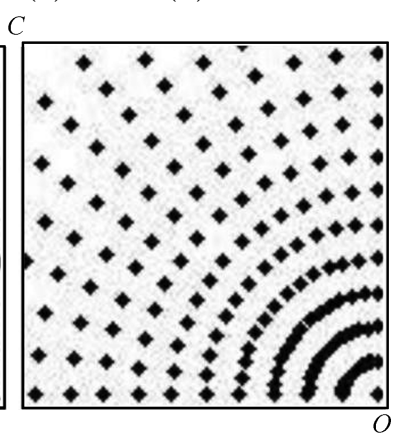

(b) Quadrant 2
Fig. 2 Projections in the first two quadrants in polar domain

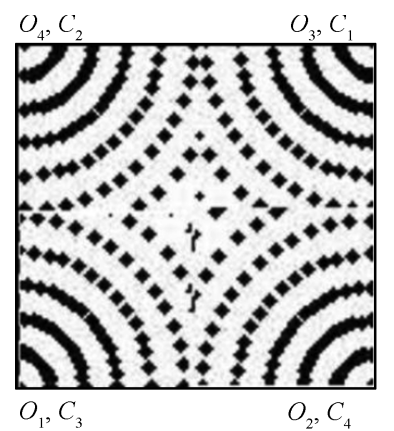

(a) Projection in all 4 quadrants for 4QFRIT

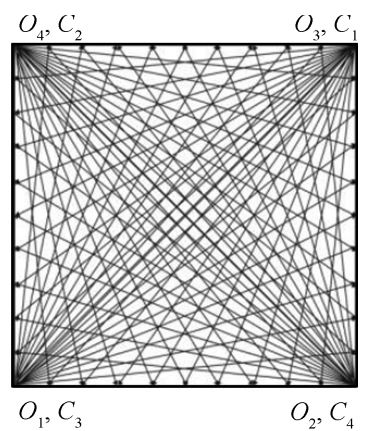

(b) Projection lines in all 4 quadrants for 4QFRIT
Fig. 3 More uniform representation achieved in 4 QFRIT

Thus, to solve the problem of non-uniform representation over a tile to represent the curves more smoothly, we propose 4-quadrant finite ridgelet transform instead of the conventional FRIT based on radon projection only in the first quadrant. Corresponding to the 4 quadrants, we take 4 sets of projections over tile $Z_{p}^{2}$. The projections for each quadrant can be written as (15). Finally, (16) presents the averaged set of projection and the 4QFRIT.

The average set of projections of each tile is used to compute its inverse ridgelet for reconstruction of the denoised 
image.

$$
\begin{aligned}
F B P_{r}(i, j) & =\frac{1}{P} \sum_{(k, 1) \in P_{i, j}} \sum_{\left(i^{\prime}, j^{\prime}\right) \in L_{k, l}} f\left[i^{\prime}, j^{\prime}\right] \\
F B P_{r}(i, j)_{m} & =\frac{1}{4 P} \sum_{m=0}^{3} \sum_{(k, l) \in P_{i, j}} \sum_{\left(i^{\prime}, j^{\prime}\right) \in L_{k, l}} f_{m}\left[i^{\prime}, j^{\prime}\right] .
\end{aligned}
$$

The indices values of $m$ (0 to 3 ) indicate the quadrants $Q_{1}$ to $Q_{4}$ respectively for computing projections of a tile $f\left[i^{\prime}, j^{\prime}\right]$.

Images can be processed using tiles of various prime number square sizes as discussed earlier. Each tile has $Z_{p}^{2}$ slopes and those slopes represent $Z_{p}^{2}$ finite projections. For denoising algorithms, the smallest tile size $3 \times 3$ is found to offer the best denoising performance. As the tile size goes on increasing, the computational load also increases, but the denoising performance marginally degrades. While computing the inverse ridgelet, there are two options: 1) The average of the four projections can be considered as resulting projection and will be subjected to the inverse ridgelet algorithm using (15). 2) The four projections as in (16) can be individually subjected to the inverse ridgelet algorithm, and the resulting four images can be averaged. In this work, we have preferred to implement the first approach. Both of them yield nearly the same results. Thus, due to the comparatively uniform distribution of projections over a tile as in Fig. 3 (b) and subsequent more uniform representation of pixels all over a tile in projection domain, the 4-quadrant ridgelet transform performs better as compared to ridgelet transform. This reduces the artifacts in the opposite corner regions to the origin of the quadrants. However, it must be noted that the improvement is at the cost of the increased computational load which increases roughly 4 times using the first option discussed above. Thus, the 4-quadrant ridgelet transform improves its denoising capability by taking average of the 4 sets of projections. The conventional hard thresholding and averaging techniques may further be used with the 4-quadrant ridgelet transform for actual denoising of the signal followed by FRIT. The other properties of the proposed 4-quadrant ridgelet transform are similar to the ridgelet transform ${ }^{[29-31]}$. Computation of 4 quadrant ridgelet transform and denoising steps of the algorithm for computation of the 4-quadrant ridgelet transform are summarized in Algorithm 3.

Algorithm 3. Denoising using 4-quadrant ridgelet transform

Step 1. Each high frequency sub-band is smoothly windowed using a window $W_{Q}$ into $m$ square tiles titled $Q_{1}$, $Q_{2}, \cdots, Q_{m}$ of appropriate size as in (8). The square window must have prime number size.

Step 2. For each tile, compute ridgelet transform using Algorithms 1 in all the 4 quadrants.

Step 3. Find average FRIT of each tile from Step 2, and insert it back in the sub-band image matrix in the place of the tile.

Step 4. Repeat Steps 2 and 3 for all the $m$ tiles.

Step 5. After computation of the 4-quadrant ridgelet transform, local averaging (low pass filtering) of the coefficients over the complete coefficient matrix using a $3 \times 3$ window is implemented.
Step 6. After local averaging, inverse FRIT is computed for achieving denoising. Inverse FRIT computation is presented as Algorithm 2.

\section{4-quadrant curvelet transform}

A 4-quadrant curvelet transform is based on band pass filtering operation followed by conditional application of 4quadrant ridgelet transform. The band pass operation isolates different scales like ridges and curves which may occur at any location with any orientation. Fig. 4 shows the flow graph of a 4-quadrant curvelet transform. It should be noted that the transform is made of an invertible chain of steps and thus theoretically has exact reconstruction property.

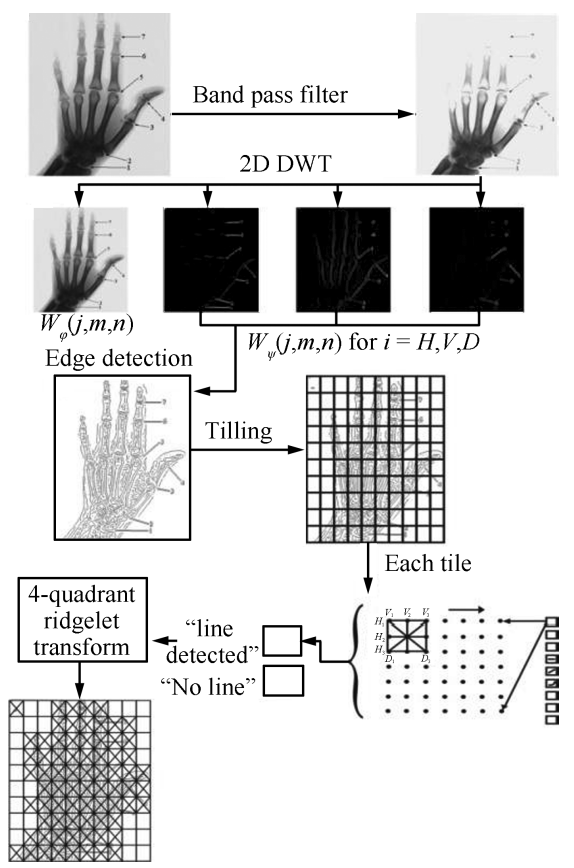

Fig. 4 Flowgraph of a 4-quadrant curvelet transform

Fig. 4 illustrates the decomposition of the band pass filtered image into sub-bands using 2D DWT to detect the edges in an image. The image is partitioned into prime size square blocks (tiles) as discussed earlier. Every tile is checked for appearance of a curved edge in it. The 4quadrant ridgelet transform is then applied to the tile if an edge exists in it. The 4QFCT computation algorithm is presented in terms of the following 4 steps.

Step 1. Band pass filter is applied on the image to compute the required 4 bands for applying $4 \mathrm{QFCT}$.

The band pass filter is implemented as a combination of a low pass filter and a high pass filter in 2D DWT. In this work, we used a perfect reconstruction Harr filter pair of 2 tap low pass and high pass coefficients, i.e., $\left[\begin{array}{ll}1 & 1\end{array}\right]$ and $[1$ $-1]$, respectively. Harr low pass filter is convolved row-wise and then column-wise, the resulting image is then convolved with the high pass filter in the same way as low pass filter to yield the band pass filtered images.

Step 2. Edge (curve) detection is carried out in the 3 high frequency bands as indicated in Fig. 4 and subse- 
quently over the main image using different thresholds discussed further.

Edge detection of nontrivial images is one of the most difficult task in image processing. Detection accuracy determines the eventual success or failure of computerized analysis procedure. We have used the following four components of 2D DWT for a threshold based edge detector in this work.

1) Average component is detected by the LPLP (low pass filter along rows and columns both) subband $W \phi(j, m, n)$.

2) Vertical edges are detected by the HPLP (high pass along rows and low pass along columns) subband $W_{\psi}^{V}(i, j)$.

3) Horizontal edges are detected by the LPHP (low pass along rows and high pass along columns) subband $W_{\psi}^{H}(i, j)$.

4) Diagonal edges are detected by the HPHP (high pass filter along rows and columns both) subband $W_{\psi}^{D}(i, j)$.

Following thresholds in (17), (18) and (19) are proposed to detect edges in color images using DWT subbands.

1) Local thresholding: This threshold is computed for a region surrounding a tile $(m>k, n>k)$.

$$
T h_{l}=\sum_{i=1}^{n} \sum_{j=1}^{m} \frac{W_{\psi}^{D}(i, j)+W_{\psi}^{H}(i, j)}{3} .
$$

2) Global thresholding: A single threshold is computed for a color band using all three frequency bands.

$$
\begin{aligned}
& T h_{g}= \\
& \frac{\sum_{i=1}^{n} \sum_{j=1}^{m} \frac{W_{\psi}^{D}(i, j)}{m \times n}+\sum_{i=1}^{n} \sum_{j=1}^{m} \frac{W_{\psi}^{V}(i, j)}{m \times n}+\sum_{i=1}^{n} \sum_{j=1}^{m} \frac{W_{\psi}^{H}(i, j)}{m \times n}}{3} .
\end{aligned}
$$

3) Mask thresholding: This is computed for a single tile at a time and is applicable only for the tile.

$$
\begin{aligned}
& T h_{m}= \\
& \frac{\sum_{i=1}^{k} \sum_{j=1}^{k} \frac{W_{\psi}^{D}(i, j)}{k^{2}}+\sum_{i=1}^{k} \sum_{j=1}^{k} \frac{W_{\psi}^{V}(i, j)}{k^{2}}+\sum_{i=1}^{k} \sum_{j=1}^{k} \frac{W_{\psi}^{H}(i, j)}{k^{2}}}{3}
\end{aligned}
$$

where $k \leqslant Z_{p}$, and $Z_{p}$ is a prime number representing a square tile side length.

The global threshold is computed only once for a complete image, or three global thresholds can be computed for the three color planes of an image using (15). The local threshold can be computed for each pixel in the sub-band and used for edge detection only at that position. The threshold for an image tile can be computed for its specific size and used for edge detection only over the tile area of the image. The computed thresholds are used for edge detection with the sub-band coefficients using simple comparison. If a coefficient value is greater than the neighboring coefficient $\left(E(i, j)=W_{\psi}^{V}(i, j)-W(i \pm 1, j \pm 1)>T h_{l, g, m}\right)$ by at least the value of threshold, then an edge is considered to present at the neighboring pixel, else the edge is absent. The edge's present spatial position is marked white (255), while the edge's absent position is marked black $(0)$.

$$
E(i, j)= \begin{cases}255, & \text { if } E(i, j)>T h_{l, g, m} \\ 0, & \text { otherwise. }\end{cases}
$$

Thus, the complete image is edge detected using one of the three thresholds. The resulting binary image $E(i, j)$ is as shown in Fig. 4. As far as this work is concerned, global threshold is used since it can give the best result. The edges of interest appear more clearly with this threshold.

Step 3. Tiling is done over the complete image.

The image is then partitioned into square tiles of prime size. In this work, we have experimented with tile sizes of $3 \times 3$ and $5 \times 5$. Each tile is checked for availability of edges in it. When we partition each sub-band into squares or tiles, we see either an "empty square" or "line detected square". The ridge fragments are nearly straight at fine scales, because an edge is nearly straight at fine scales. Such nearly straight ridge fragments are the desired inputs for the 4 quadrant ridgelet transform.

Step 4. 4-quadrant ridgelet transform as in (15) and (16) is applied only to the tiles in which an edge exists.

The inverse procedure is exactly opposite to these four steps, i.e., it starts at Step 4 and ends at Step 1. The inverse ridgelet is applied only on tiles containing edges. The order of application of low pass and high pass filters is also reversed. A record of tiles on which the 4QFRIT is applied is maintained for computing their inverse 4QFRIT.

\section{Experimental results and discussion}

The experimentation has been carried out on a variety of gray scale and color images of different types. However, only a few representative results have been presented here.

The 2D DWT, FRIT, FCT and 4QFCT were implemented for benchmarking of the denoising and curves processing performance. Initially, Table 1 presents MSE and PSNR results of transforms and the subsequent reconstructions of the original test images. The term "method noise" represents the intrinsic noise introduced in an original image by a denoising algorithm in absence of any external noise or zero external noise. An ideal denoising algorithm should have zero method noise. Thus, Table 1 represents method noise of the transforms. The test images were further added Gaussian noise of varying means $(\mu=2$ to 12$)$ and standard deviation ( $\sigma=2$ to 10). Denoising performance of the algorithms in presence of noise $(\mu=0, \sigma=2)$ is the same as that in presence of noise $(\mu=2, \sigma=2)$. The resulting noisy images were denoised using the said transforms, and benchmarked using MSE and PSNR of the individual reconstructed images. The comparative denoising performance has been presented in Table 2. It is clear from Table 2 that the proposed $4 \mathrm{QFCT}$ yields on an average $7 \mathrm{~dB}$ to $8 \mathrm{~dB}$ better performance than 2D DWT, around $3 \mathrm{~dB}$ to $4 \mathrm{~dB}$ better performance than the FRIT, $0.75 \mathrm{~dB}$ to $1 \mathrm{~dB}$ better performance than the FCT. However, the curve processing performance is not obvious from the denoising performance. Visual comparison of images is the only way to compare the curve processing performance. The visual denoising results are presented on a gray X-ray wristhand image in Figs. $5(\mathrm{a})-5(\mathrm{~h})$. Figs. 5 (a) presents original wristhand image. Figs. $5(\mathrm{~b})-5$ (d) present the reconstructed images using FRIT, FCT and 4QFCT. Fig. 5 (e) presents the gaussian noise $(\mu=10, \sigma=12)$ added image. Figs. $5(\mathrm{f})-5(\mathrm{~h})$ present the denoised images using FRIT, FCT and 4QFCT.

Note the smooth reconstruction of the denoised image in Fig. $5(\mathrm{~h})$ and specially the numbers compared to all the other reconstructed images. 
Table 1 Reconstruction using the transforms represents method noise

\begin{tabular}{ccccccccc}
\hline Images & \multicolumn{2}{c}{$\begin{array}{c}\text { 2D wavelet } \\
\text { transform }\end{array}$} & \multicolumn{2}{c}{$\begin{array}{c}\text { Finite ridgelet } \\
\text { transform }\end{array}$} & \multicolumn{2}{c}{$\begin{array}{c}\text { Finite curvelet } \\
\text { transform }\end{array}$} & \multicolumn{2}{c}{$\begin{array}{c}\text { 4-quadrant curvelet } \\
\text { transform }\end{array}$} \\
& MSE & PSNR (dB) & MSE & PSNR (dB) & MSE & PSNR (dB) & MSE & PSNR (dB) \\
\hline Barbara & 56.8 & 30.6 & 56.7 & 30.6 & 54.1 & 30.8 & 34.5 & 32.7 \\
Wristhand & 25.5 & 34.6 & 14.7 & 36.5 & 6.4 & 40.1 & 5.8 & 40.8 \\
Peppers & 28.8 & 33.6 & 17.8 & 35.7 & 10.4 & 37.9 & 8.2 & 38.9 \\
Severe 2 & 14.5 & 36.5 & 10 & 38.1 & 2.9 & 42.4 & 2.6 & 43.9 \\
\hline
\end{tabular}

Table 2 Denoising performance of various transforms

\begin{tabular}{|c|c|c|c|c|c|c|c|c|c|}
\hline \multirow{2}{*}{$I^{*}$} & \multirow{2}{*}{ Noise $\mu, \sigma$} & \multicolumn{2}{|c|}{ DWT } & \multicolumn{2}{|c|}{ FRIT } & \multicolumn{2}{|c|}{$\mathrm{FCT}$} & \multicolumn{2}{|c|}{$4 \mathrm{QFCT}$} \\
\hline & & MSE & PSNR (dB) & MSE & PSNR (dB) & MSE & PSNR (dB) & MSE & PSNR (dB) \\
\hline \multirow[t]{5}{*}{$1^{*}$} & 2,2 & 27.1 & 33.8 & 14.9 & 36.4 & 6.8 & 39.8 & 5.7 & 40.5 \\
\hline & 4,4 & 34.1 & 32.8 & 19.1 & 35.3 & 7.3 & 39.4 & 6.1 & 40.2 \\
\hline & 7,5 & 41 & 32 & 25.2 & 34.1 & 8 & 39.1 & 6.7 & 39.8 \\
\hline & 9,7 & 46 & 31.5 & 27.1 & 33.8 & 9.8 & 38.2 & 8.2 & 38.9 \\
\hline & 12,10 & 47.1 & 31.4 & 32.5 & 33 & 11.0 & 37.5 & 10.3 & 37.9 \\
\hline \multirow[t]{5}{*}{$2^{*}$} & 2,2 & 74.6 & 29.4 & 63.5 & 30.1 & 56.6 & 30.6 & 38.2 & 32.3 \\
\hline & 4,4 & 85.7 & 28.8 & 74.6 & 29.4 & 63.5 & 30.1 & 42.9 & 31.8 \\
\hline & 7,5 & 96.1 & 28.3 & 83.7 & 28.9 & 69.6 & 29.7 & 50.4 & 31.1 \\
\hline & 9,7 & 105.4 & 27.9 & 98.4 & 28.2 & 81.8 & 29 & 55.3 & 30.7 \\
\hline & 12,10 & 113 & 27.6 & 105.4 & 27.9 & 89.7 & 28.6 & 62.1 & 30.2 \\
\hline \multirow[t]{5}{*}{$3^{*}$} & 2,2 & 37 & 32.4 & 20.6 & 34.9 & 10.8 & 37.9 & 8.2 & 38.9 \\
\hline & 4,4 & 34.2 & 32.8 & 24.3 & 34.2 & 10.4 & 37.7 & 8.7 & 38.7 \\
\hline & 7,5 & 49.8 & 31.2 & 26.8 & 33.8 & 12.3 & 37.2 & 9.7 & 38.2 \\
\hline & 9,7 & 71 & 29.6 & 30.9 & 33.2 & 13.1 & 36.9 & 10.3 & 37.9 \\
\hline & 12,10 & 79.8 & 29.1 & 36.1 & 32.5 & 14.9 & 36.4 & 10.7 & 37.8 \\
\hline \multirow[t]{5}{*}{$4^{*}$} & 2,2 & 105.4 & 27.9 & 78.1 & 29.2 & 5.4 & 40.5 & 3.9 & 42.2 \\
\hline & 4,4 & 159.6 & 26.1 & 110.4 & 27.7 & 8.8 & 38.5 & 7.9 & 39.1 \\
\hline & 7,5 & 183.2 & 25.5 & 149 & 26.4 & 17.3 & 35.7 & 14.2 & 36.5 \\
\hline & 9,7 & 241.5 & 24.3 & 201 & 25.1 & 27.8 & 33.6 & 24.7 & 34.2 \\
\hline & 12,10 & 253 & 24.1 & 187.5 & 25.4 & 34.7 & 32.7 & 28.6 & 33.5 \\
\hline
\end{tabular}

$\mathrm{I}^{*}$ : Images, $1^{*}$ : Wristhand, $2^{*}$ : Barbara, $3^{*}$ : Peppers, $4^{*}$ : Severe 2.

Fig. 6 (a) presents a close up portion of the original image in Fig. 5 (a). Fig. 6 (b) presents the reconstruction using 4QFCT. Fig. 6 (c) presents the same portion after adding the said noise as in Fig. 5 (e).

Figs. $6(\mathrm{~d})-6$ (f) present the reconstructed images using FRIT, FCT and 4QFCT. The edges of the bones in the $\mathrm{X}$-ray image reconstructed using $4 \mathrm{QFCT}$ are least worn.

Figs. 7(a) $-7(\mathrm{~h})$ present a set of color biomedical images titled Severe 2. Fig. 7 (a) presents original image, while Figs. $7(\mathrm{~b})-7(\mathrm{~h})$ present the processed images in exactly the same sequence to the Wristhand images presented in Fig. 5 (a) 5(h). Similar observations can be made from Figs. $7(\mathrm{~b})-7(\mathrm{~h})$. The results of $4 \mathrm{QFCT}$ with and without presence of the noise $(\mu=10, \sigma=12)$ as presented in Figs. $7(\mathrm{~d})$ and $7(\mathrm{~h})$ are much better as compared to the other discussed transforms; quantitatively and visually. A small part of the original Severe 2 as in Figs. 8 (a) is enlarged and processed using the discussed algorithms. The results are presented in Figs. 8(b)-8(f). The result of denoising using 4QFCT as in Fig. 8 (f) is comparably smoother than the other results. The denoising performance in Table 2 is plotted in Figs. 9 and 10 between standard deviation of the added noise and PSNR of reconstruction for Wristhand and Severe 2 images. It has been found that PSNR decreases gradually with the increase in noise level.

The bar chart in Fig. 11 benchmarks 2D DWT, FCT with 4QFCT denoising performance on the test images. These experiments have been carried out on a P-IV , 2.6 GHz, 2 GB RAM with Windows. Matlab R2009a has been used as the computing platform.

The timing requirement and comparison for denoising of images are highly subjective and depends upon the image size, type (gray or color), machine, operating system setup, computational platform and their settings. For presenting the computational time comparison, we have executed all the algorithms on a noisy gray image of resolution $256 \times 256$. The denoising computations with $2 \mathrm{D}$ DWT takes roughly $0.8 \mathrm{~s}$. Denoising of the same image with 


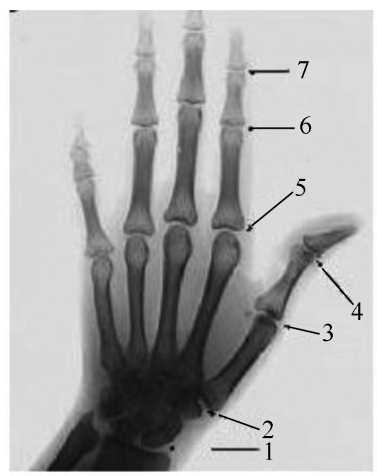

(a) Original Wristhand

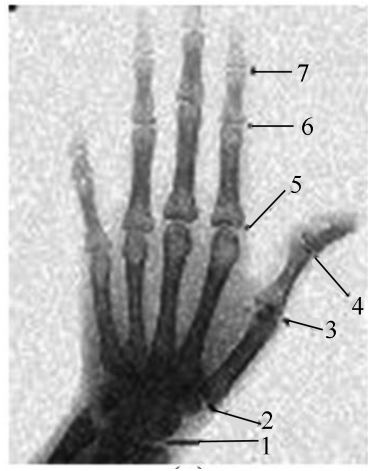

(e) Noisy

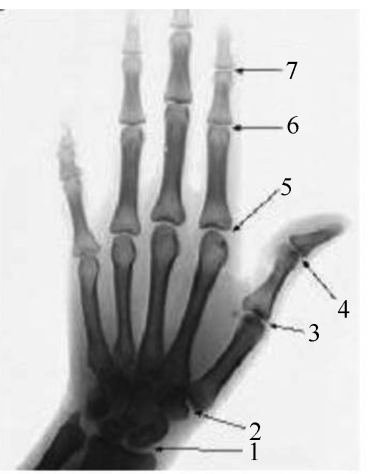

(b) Reconstructed using FRIT

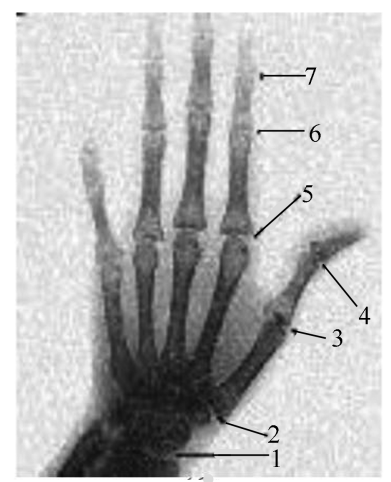

(f) Denoised using FRIT

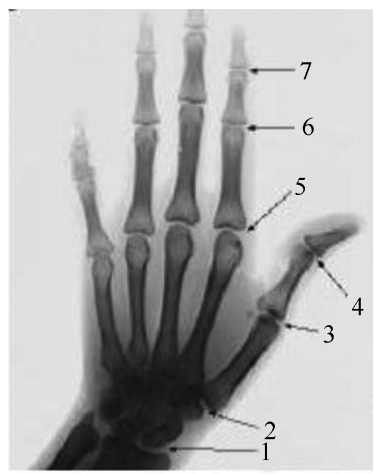

(c) Reconstructed using FCT

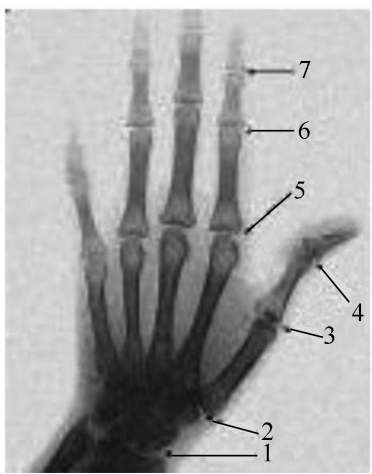

(g) Denoised using FCT

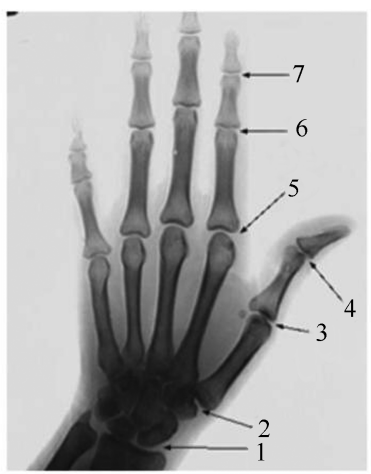

(d) Reconstructed using $4 \mathrm{QFCT}$

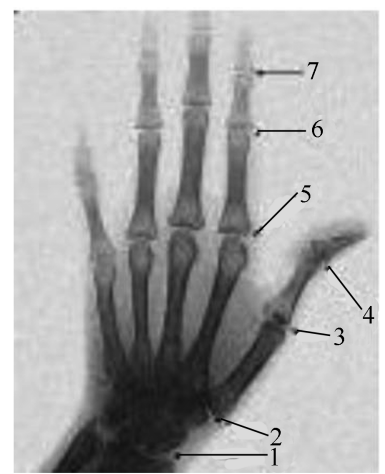

(h) Denoised using 4QFCT

Fig. 5 Wristhand: original, reconstructed and denoised images

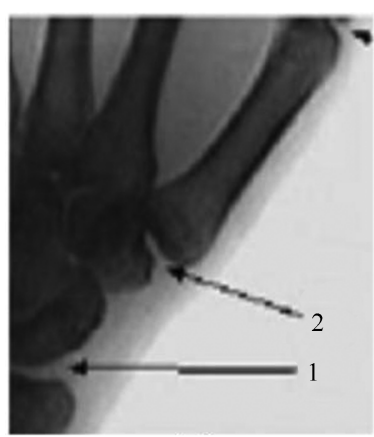

(a) Part of Wristhand

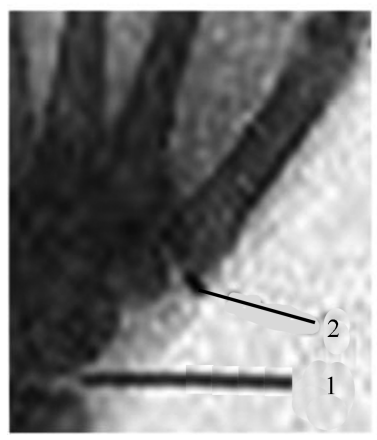

(d) Denoised using FRIT

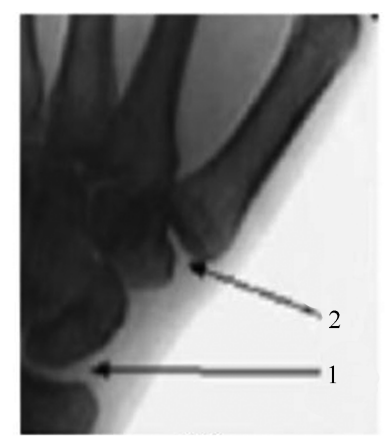

(b) Reconstructed using $4 \mathrm{QFCT}$

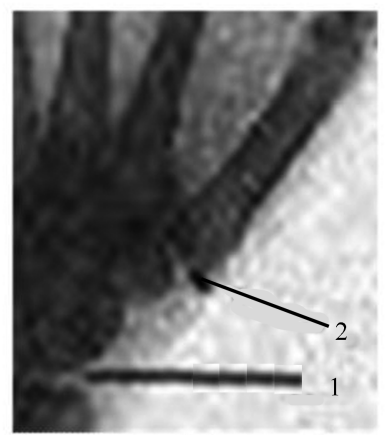

(e) Denoised using FCT

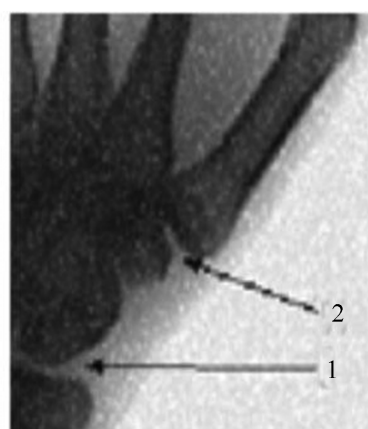

(c) Noisy

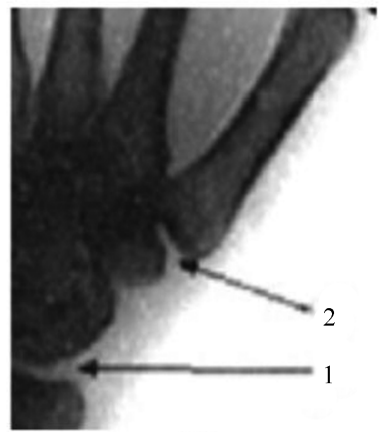

(f) Denoised using 4QFCT

Fig. 6 Part of Wristhand: original, reconstructed and denoised images 


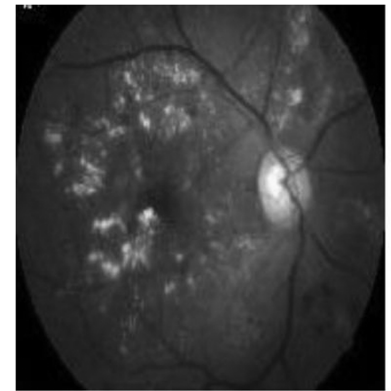

(a) Original Severe2

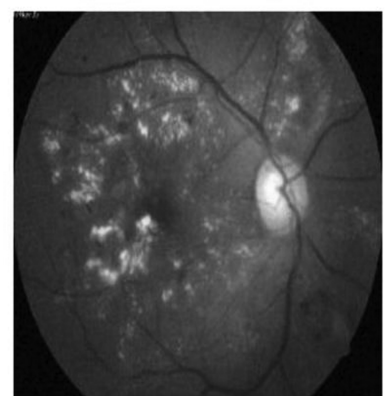

(e) Noisy

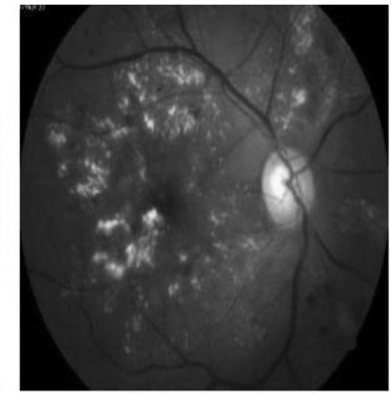

(b) Reconstructed using FRIT

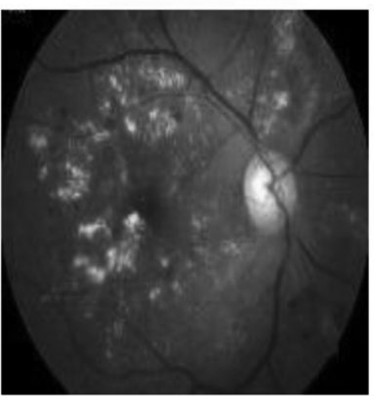

(f) Denoised using FRIT

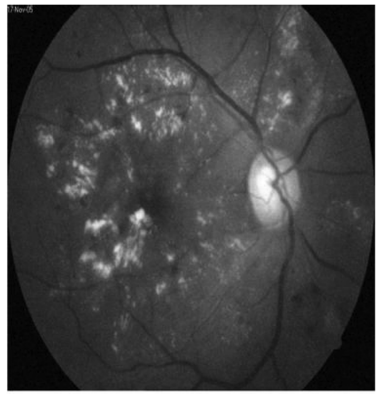

(c) Reconstructed using FCT

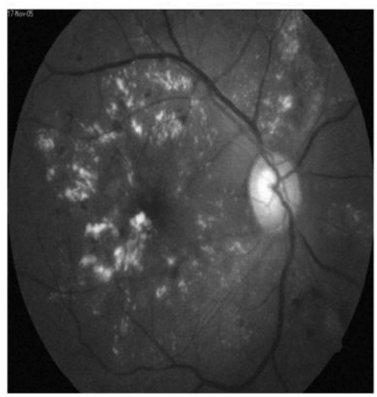

(g) Denoised using FCT

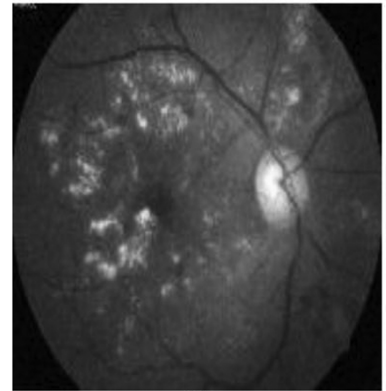

(d) Reconstructed using 4QFCT

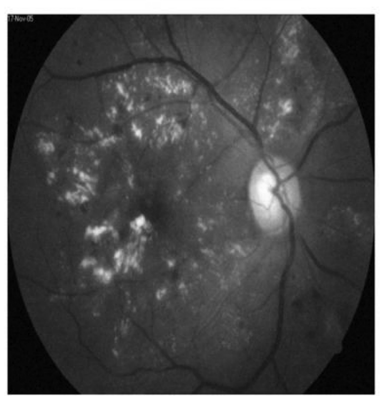

(h) Denoised using 4QFCT

Fig. 7 Severe 2: original, reconstructed and denoised images

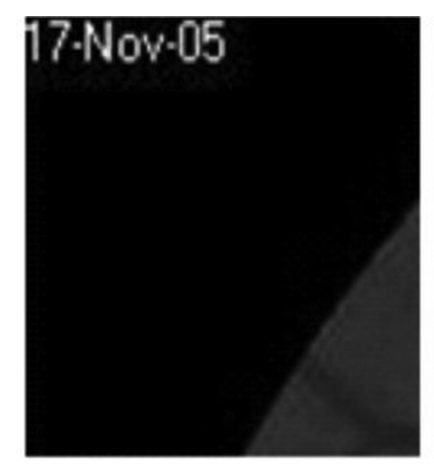

(a) Part of Severe 2

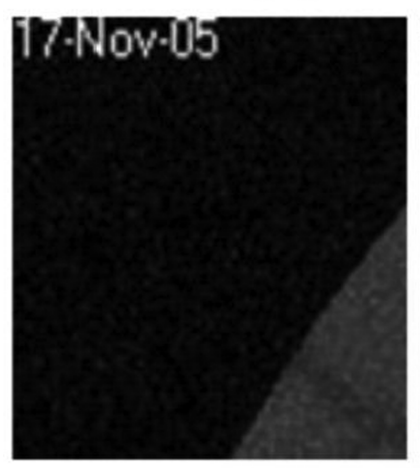

(d) Denoised using FRIT

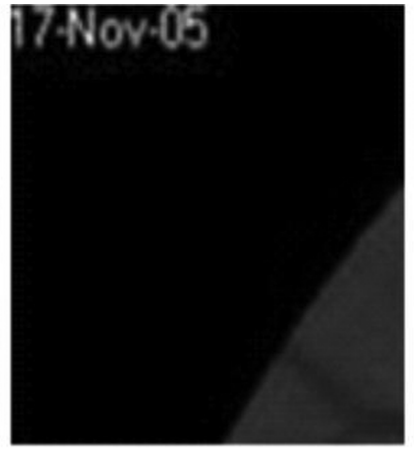

(b) Reconstructed using 4QFCT

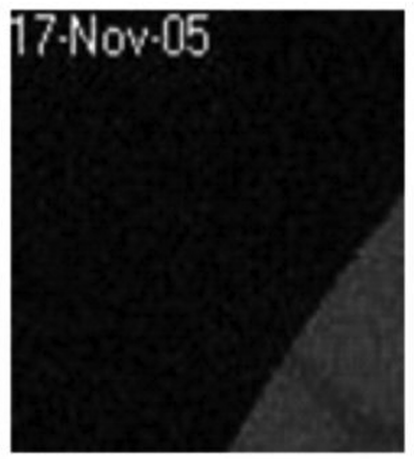

(e) Denoised using FCT

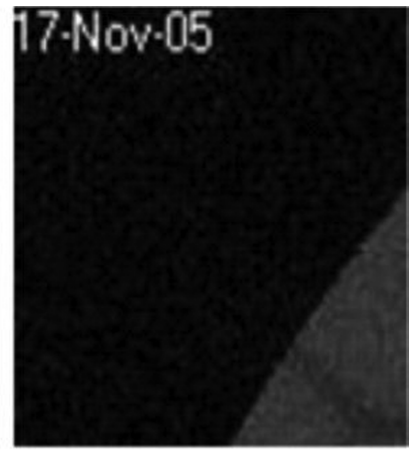

(c) Noisy

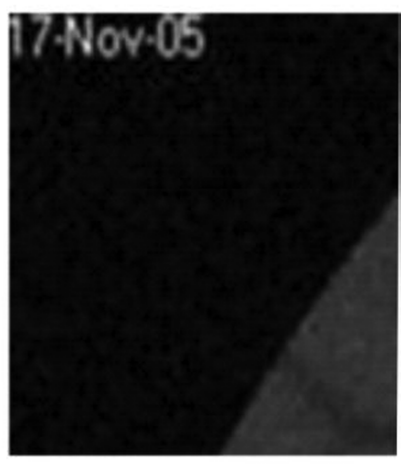

(f) Denoised using 4QFCT

Fig. 8 Part of Severe2: original, reconstructed and denoised Images 
FRIT $(3 \times 3$ tile) takes around $6 \mathrm{~s}$. Denoising using FCT requires $4 \mathrm{~s}$. Denoising using the proposed $4 \mathrm{QFCT}$ requires around $8 \mathrm{~s}$. Thus, a better denoising and smooth curves are achieved at the cost of the increased computational time.

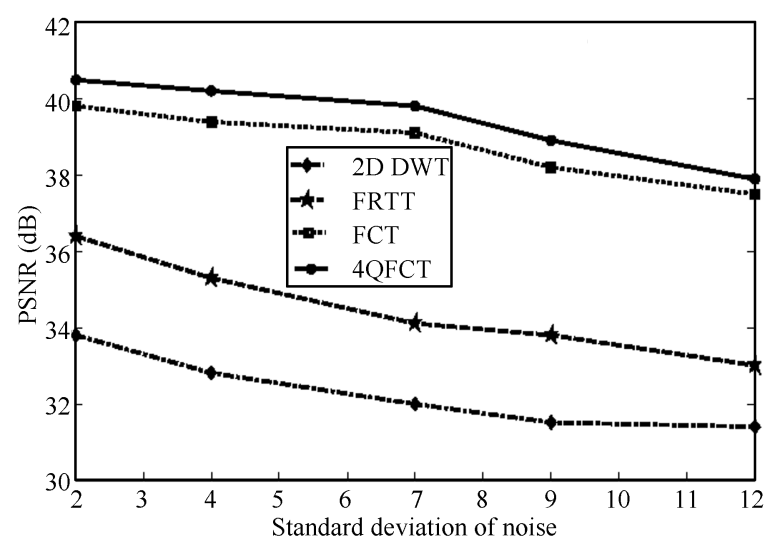

Fig. 9 PSNR in dB against standard devation of noise for Wristhand

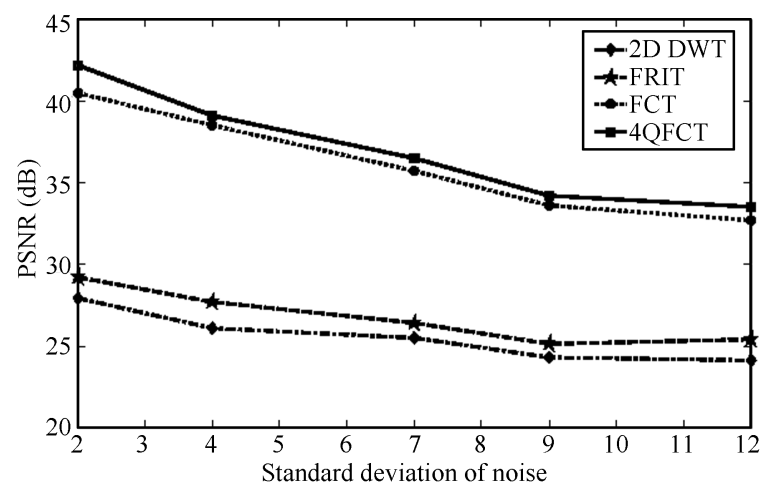

Fig. 10 PSNR in dB against standard deviation of noise for Severe 2

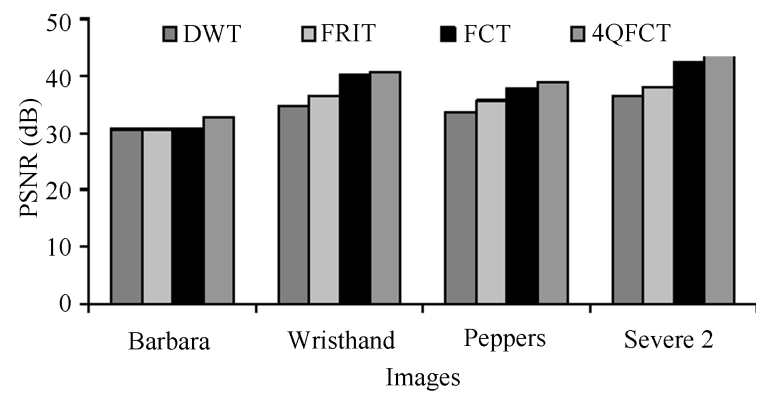

Fig. 11 Denoising performance of 2D DWT, finite curvelet transform and 4-quadrant curvelet transform

This work uses theory of angular projections to explain concepts of the poor representation of annular region pixels in large size tiles and to explain better representation of sharp curves using radial system like representation in ridgelets against the Cartesian coordinate system. We have used discrete parallel projections in actual implementation. The reconstruction of the text and numbers in the result images of the proposed algorithm are smoother than those in the results of the other discussed algorithms, though they are slightly blurred in the process of denoising. Improvement in PSNR result from thresholding of coefficients, averaging in curvelet/spatial domain and robust signal representation using 4QFRIT. Though inclusion of mean value of random noise is non-conventional in the image processing experiments, it highlights the fact that the quantitative denoising performance is only marginally dependent on the mean value even for large mean values of random noise ranging up to $10 \%$ of the peak signal. The tile sizes of $3 \times 3$ and $5 \times 5$ are found to be suitable for a broad variety of images. However, bigger tile sizes can be used for images that are not expected to contain curves with sharp turns. It is evident from the result images that the visual performance of the proposed algorithm is much better than that of the other discussed algorithms.

\section{Conclusions}

The proposed 4QFCT that uses average of the 4 quadrant radon projection improves the denoising performance and representation of smooth curves compared to FRIT and FCT. Though the proposed 4QFCT improves the denoising performance only by a marginal value of around $1 \mathrm{~dB}$ compared to FCT, it improves the performance considerably compared to FRIT on an average by around $6 \mathrm{~dB}$. Thus, though the 4QFCT yields only slight improvement in PSNR of reconstruction from noisy images compared to FCT, it yields better visual quality images at less computational cost compared to FRIT and FCT. The smallest $3 \times 3$ tile yields best denoising and curve processing performance with FRIT, FCT and 4QFCT.

\section{References}

[1] P. Rajavel. Image dependent brightness preserving histogram equalization. IEEE Transactions on Consumer Electronics, vol. 56, no. 2, pp. 756-763, 2010.

[2] C. Zhang, L. L. Cheng, Z. D. Qiu, L. M. Cheng. Multipurpose watermarking based on multiscale curvelet transform. IEEE Transactions on Information Forensics and Security, vol. 3, no. 4, pp. 611-619, 2008.

[3] M. S. Miri, A. Mahloojifar. Retinal image analysis using curvelet transform and multistructure elements morphology by reconstruction. IEEE Transactions on Biomedical Engineering, vol. 58, no. 5, pp. 1183-1192, 2011.

[4] S. S. Kumar, R. S. Moni. Diagnosis of liver tumor from CT images using curvelet transform. International Journal of Computer Science and Engineering, vol. 2, no. 4, pp. 11731178, 2010.

[5] M. Freiman, M. Werman, L. Joskowicz. A curvelet-based patient specific prior for accurate multi-modal brain image rigid registration. Medical Image Analysis, vol. 15, pp. 125$132,2011$.

[6] L. F. Wei, Y. F. Sun, B. C. Yin. Face recognition using common vector based on curvelet transform. In Proceedings of the International Multiconference of Engineers and Computer Scientist, Hong Kong, China, vol. 1, pp. 1-6, 2011.

[7] H. Hejazi, M. Alhanjouri. Face recognition using curvelet transform. In Proceedings of the 23rd International Conference on Industrial Engineering and Other Applications of Applied Intelligent Systems, Springer-Verlag, Berlin, Heidelberg, Germany, pp. 388-396, 2010. 
[8] G. J. Reddy, T. J. C. Prasad, M. N. G. Prasad. Fingerprint image denoising using curvelet transform. ARPN Journal of Engineering and Applied Sciences, vol.3, no.3, pp.31-35, 2008.

[9] M. Amirmazlaghan, H. Amindavar. Two novel Bayesian multiscale approaches for speckle suppression in SAR images. IEEE Transactions on Geoscience and Remote Sensing, vol. 48, no. 7, pp. 2980-2993, 2010.

[10] J. L. Starck, F. Murtagh, E. J. Candes, D. L. Donoho. Gray and color image contrast enhancement by the curvelet transform. IEEE Transactions on Image Processing, vol. 12, no. 6, pp. 706-717, 2003.

[11] J. L. Starck, E. J. Candes, D. L. Donoho. The curvelet transform for image denoising. IEEE Transactions on Image Processing, vol. 11, no. 6, pp.670-683, 2002.

[12] D. L. Donoho. De-noising by soft-thresholding. IEEE Transactions on Information Theory, vol. 41, no. 3, pp. 613-627, 1995.

[13] G. Hennenfent, F. J. Herrmann. Seismic denoising with nonuniformly sampled curvelets. Computing in Science \& Engineering, vol. 8, no. 3, pp. 16-25, 2006.

[14] J. W. Ma, G. Plonka. Combined curvelet shrinkage and nonlinear anisotropic diffusion. IEEE Transactions on Image Processing, vol. 16, no. 9, pp. 2198-2206, 2007.

[15] J. W. Ma. Compressed sensing for surface characterization and metrology. IEEE Transactions on Instrumentation and Measurement, vol. 59, no. 6, pp. 1600-1615, 2010.

[16] J. W. Ma. Single-pixel remote sensing. IEEE Geoscience and Remote Sensing Letters, vol. 6, no. 2, pp. 199-203, 2009.

[17] D. L. Li, Z. M. Duan, M. Jia. New method based on curvelet transform for image denoising. In Proceedings of the IEEE International Conference on Measuring Technology and Mechatronics Automation, IEEE, Changsha, China, pp. 760-763, 2010.

[18] J. W. Ma, G. Plonka. Computing with curvelets: From image processing to turbulent flows. Computing in Science and Engineering, vol. 11, no. 2, pp. 72-80, 2009

[19] G. Tang, J. W. Ma. Application of total-variation-based curvelet shrinkage for three dimensional seismic data denoising. IEEE Geoscience and Remote Sensing Letters, vol. 8, no. 1, pp. 103-107, 2011.

[20] G. R. Easley, D. Labate, F. Colonna. Shearlet-based total variation diffusion for denoising. IEEE Transactions on Image Processing, vol. 18, no. 2, pp. 260-268, 2009.

[21] T. Jiang, X. Zhao. Research and application of image denoising method based on curvelet transform. In Proceedings of the International Archives of the Photogrammetry, Remote Sensing and Spatial Information Sciences, Beijing, China, vo1. XXXVII, Part B2, pp. 363-367, 2008.

[22] H. Rabbani, S. Gazor. Image denoising employing local mixture models in sparse domains. IET Image Processing, vol. 4, no. 5, pp. 413-428, 2010.

[23] I. J. Sumana, M. M. Islam, D. S. Zhang, G. J. Lu. Content based image retrieval using curvelet transform. In Proceedings of the 10th IEEE Workshop on Multimedia Signal, IEEE, Cairns, Qld, Australia, pp. 11-16, 2008.

[24] D. L. Donoho, M. R. Duncan. Digital curvelet transform: Strategy, implementation and experiments. In Proceedings of SPIE, vol. 4056, pp. 12-29, 2000.
[25] L. Dettori, L. Semler. A comparison of wavelet, ridgelet, and curvelet-based texture classification algorithms in computed tomography. Computers in Biology and Medicine, vol. 37, no. 4, pp. 486-498, 2007.

[26] J. L. Stuck, M. Elad, D. L. Donoho. Image decomposition via the combination of sparse representations and a variational approach. IEEE Transactions on Image Processing, vol. 14. no. 10, pp. 1570-1582, 2005.

[27] S. Chandra, N. Normand, A. Kingston, J. Guedon, I. Svalbe. Fast Mojette transform for discrete tomography, [Online], Available: http://arxiv.org/abs/1006.1965v1, June 11, 2010.

[28] I. Svalbe, A. Kingston. Farey sequences and discrete radon transform projection angles. Electronics Notes in Discrete Mathematics, vol. 12, pp. 154-165, 2003.

[29] 29. G. Y. Chen, B. Kegl. Complex ridgelets for image denoising. Pattern Recognition, vol. 40, pp. 578-585, 2007.

[30] M. N. Do, M. Vetterli. Orthonormal finite ridgelet transform for image compression. In Proceedings of IEEE International Conference on Image Processing, IEEE, Vancouver, BC, Canada, vol. 2, pp. 367-370, 2000

[31] M. N. Do, M. Vetterli. The finite ridgelet transform for image representation. IEEE Transactions on Image Processing, vol. 12, no. 1, pp. 16-28, 2003.

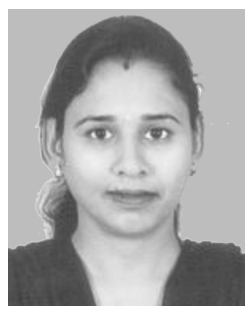

P. K. Parlewar received her B. Eng. degree from Government College of Engineering, India in 2000, received her M. Tech. and $\mathrm{Ph}$. D. degrees from Nagpur University, India in 2006 and 2013. She is currently an assistant professor with Shri Ramdeobaba College of Engineering and Management, India.

Her research interests include image processing, computer vision, transform based denoising techniques, and analog devices and circuits.

E-mail: pallaviparlewar@rknec.edu

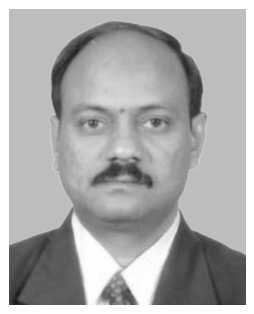

K. M. Bhurchandi received his B. Eng. and M. Eng. degrees in electronics engineering in 1990 and 1992. He further obtained his $\mathrm{Ph} . \mathrm{D}$. degree from Visvesvaraya Regional College of Engineering, Nagpur University, Nagpur, India in 2002, where he is currently working as an associate professor. He is principal investigator of a funded research project titled "Scale Invariant Face Recognition using Gray Images. He has more than 45 publications to his credit". He has also worked on industrial problems on heavy and in motion weighing systems. He is the co-author of a popular book titled Advanced Microprocessors and Peripherals published by McGraw Hill, India.

His research interests include color image processing and analysis, computer vision, digital signal processing, embedded systems and microprocessor architectures.

E-mail: bhurchandikm@yahoo.co.in 